\title{
All bent out of shape: buckling of sheared fluid layers
}

\author{
Neil M. Ribe $\dagger$
}

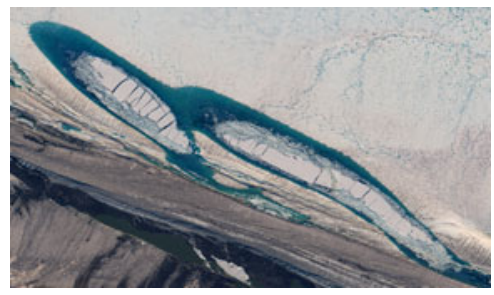

Laboratoire FAST, UPMC/Univ Paris-Sud/CNRS, Bât. 502,

Campus Universitaire, 91405 Orsay, France

Buckling instabilities of thin sheets or plates of viscous fluid occur in situations ranging from food and polymer processing to geology. Slim, Teichman \& Mahadevan (J. Fluid Mech., this issue, vol. 694, 2012, pp. 5-28) study numerically the buckling of a sheared viscous plate floating on a denser fluid using three approaches: a classical 'thin viscous plate' model; full numerical solution of the three-dimensional Stokes equations; and a novel 'advection-augmented' thin-plate model that accounts (in an asymptotically inconsistent way) for the advection of perturbations by the background shear flow. The advection-augmented thin-plate model is markedly superior to the classical one in its ability to reproduce the predictions of the Stokes solution, illustrating the utility of judicious violations of asymptotic consistency in fluidmechanical models.

\section{Introduction}

Among the most beautiful structures in fluid mechanics are those produced by the buckling of thin fluid layers in response to external compression. Travellers in mountainous regions marvel at the complex folded structures produced by the slow buckling of ductile rock strata over geological time scales (Johnson \& Fletcher 1994). The figure by the title (S. Niebuhr \& P. Morin, Polar Geospatial Center, copyright DigitalGlobe, Inc., used with permission) shows a satellite image of ice-shelf surface undulations on the George VI Ice Shelf along its boundary with the rocky coast of Alexander Island, Antarctica. Depressed portions of the ice shelf fill with surface meltwater to form pear-shaped, 'en échelon' lakes. The lakes are also covered with thin floating lake ice that is variously fragmented, and are separated by upward arching rolls on the ice shelf. Ice flows obliquely towards the coast (i.e. from upper right to lower left) with speed $v_{I} \lesssim 50 \mathrm{~m} \mathrm{year}^{-1}$. The undulations, and meltwater lakes they trap, propagate along the coast (from lower right to upper left) at a speed $v_{U}>10 v_{I}$, as an apparent consequence of viscous buckling of the ice shelf (LaBarbera \& MacAyeal 2012). A more familiar example much closer to home is the periodic folding instability of a vertical sheet of viscous fluid (e.g. cake batter) falling onto a surface (Skorobogatiy \& Mahadevan 2000; Ribe 2003).

$\dagger$ Email address for correspondence: ribe@ fast.u-psud.fr 
Buckling viscous sheets are thin objects with one small dimension (the thickness) and two much larger dimensions, and can moreover experience large changes of shape. The dynamics of thin deformable sheets are described by a branch of mechanics known as shell theory, which is highly developed for elastic sheets (Niordson 1985). Derivations of the analogous equations for viscous sheets include those of Buckmaster, Nachman \& Ting (1975), Howell (1996), and Ribe (2002).

The essential physical content of shell theory is that a thin sheet can deform either by stretching or by bending, depending on its shape and the distribution of the external forces acting on it. Buckling instabilities are dominated by bending, whose defining characteristic is a linear variation of the midsurface-parallel fibre stress $\sigma_{s s}(z)$ across the sheet's thickness, from compressional $\left(\sigma_{s s}<0\right)$ on the side towards the centre of bending to extensional on the other side. For a two-dimensional viscous sheet with viscosity $\mu, \sigma_{s s}=-4 \mu \dot{K} z$, where $z=0$ is the sheet's midsurface and $\dot{K}$ is the rate of change of the curvature $K$ of that surface. The first moment of this stress distribution for a sheet of thickness $h$ is the bending moment $M=-\mu h^{3} \dot{K} / 3$, which shows that the sheet resists bending as the cube of its thickness.

Buckling instabilities are governed by the balance of forces acting on the sheet in the direction normal to the midsurface. With gravity and surface tension neglected for simplicity, this balance is $\partial_{s s}^{2} M+K N=0$, where $N$ is the extensional $(N>0)$ or compressional $(N<0)$ membrane force acting parallel to the midsurface. Let the deflection of the midsurface from an initially flat configuration be $H(s, t)=$ $H_{0} \sin k s \exp \sigma t$, where $\sigma$ is the growth rate. With $K=\partial_{s s}^{2} H$ and $\dot{K}=\partial_{t} \partial_{s s}^{2} H$, the force balance implies $\sigma=-3 N / \mu h^{3} k^{2}$, which is positive only if $N<0$. Buckling is therefore possible only if the sheet is compressed along its midsurface.

\section{Overview}

Several authors (Taylor 1969; Suleiman \& Munson 1981; Benjamin \& Mullin 1988) have performed laboratory experiments on buckling of a sheared floating plate of viscous fluid in the form of a circular annulus, in the configuration shown schematically in figure 1(a). As reported in the following paper, Slim, Teichman \& Mahadevan (2012) study a simpler model in which the plate is infinitely long, as shown in figure $1(b)$. The motion of the bounding walls at speeds $\pm U$ generates a linear shear velocity profile (brown arrows) that compresses material elements in a direction inclined at $45^{\circ}$ to the walls (black arrows), leading to buckling.

Benjamin \& Mullin (1988) analysed the buckling of a laterally infinite viscous plate with uniform background shear using both a thin-plate model and a complete analytical solution of the three-dimensional Stokes equations. Slim et al. (2012) improve on their work in two ways. First, they include the influence of the bounding walls, which constrain the form the buckling can take. Second, they propose a new 'advection-augmented' thin-plate model that includes advection of perturbations by the background flow, and they compare its predictions with those of the simple thin-plate model and the Stokes solution.

Slim et al.'s important result is that the advection-augmented plate model reproduces the predictions of the Stokes solution much better than does the simple plate model without advection. The advection-augmented model comprises a pair of partial differential equations for the vertical deflection $H$ and the vertical velocity $w$ of the midsurface:

$$
\begin{gathered}
-2 \mu h S \partial_{x y}^{2} H=-\frac{1}{3} \mu h^{3} \nabla^{4} w+\left(\gamma^{+}+\gamma^{-}\right) \nabla^{2} H-\left(\rho^{-}-\rho_{\text {air }}\right) g H, \\
w=\partial_{t} H+(S y) \partial_{x} H,
\end{gathered}
$$


(a)

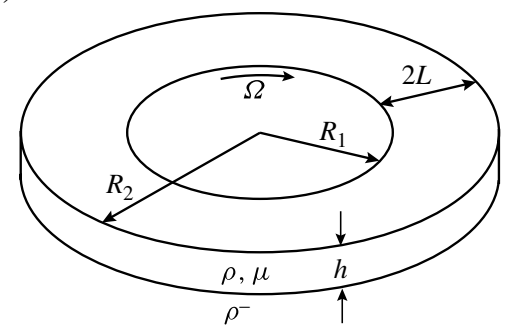

(b)

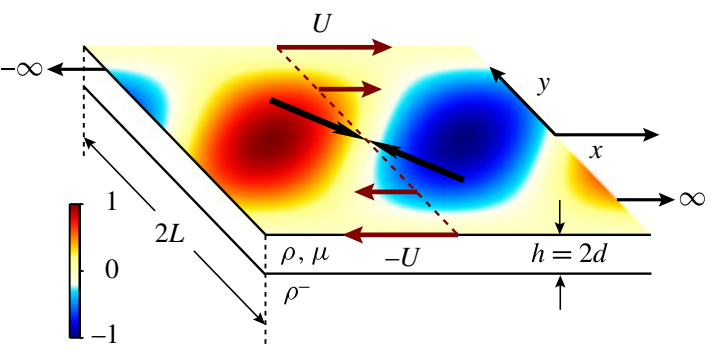

FIGURE 1. Configurations used to study buckling of thin sheared viscous plates. (a) Annular geometry typically used in laboratory experiments (Taylor 1969; Suleiman \& Munson 1981; Benjamin \& Mullin 1988), in which shear is induced by steady rotation of one bounding wall with angular speed $\Omega$. (b) Configuration of Slim et al. (2012), in which parallel bounding walls move at speeds $\pm U$. The background shear velocity profile is shown by brown arrows, and the direction of the maximum rate of compression by black arrows. Colours indicate the amplitude (in arbitrary units) of the most unstable buckling mode for $d / L=0.02$ and $\gamma L / d \mu U=0.3$, where $\gamma$ is the surface tension coefficient (assumed to be the same on both surfaces of the plate).

where $S=U / L$ is the shear rate. The equations of the simple plate model are the same as (2.1) except that the advection term $(S y) \partial_{x} H$ in $(2.1 b)$ is absent.

Equation (2.1a) expresses the balance of vertical forces acting on the plate. The left-hand side is the vertical component of the background membrane stress acting along the curved midsurface, and is the driving force for buckling. It is balanced by three resisting forces on the right-hand side: the viscous resistance to bending (first term), surface tension acting on the plate's upper $\left(^{+}\right)$and lower $\left(^{-}\right)$surfaces (second term), and the restoring force of buoyancy (third term), which is proportional to the difference of the densities of the fluids below and above the plate.

When the stabilizing effects of surface tension and buoyancy are absent, perturbations of all wavelengths are unstable for any shear rate. But because viscous bending resistance slows the growth of short wavelengths while the bounding walls slow the growth of long ones, the fastest growth occurs at an intermediate wavelength $\lambda \approx 3.32 L$. The corresponding stationary buckling mode spans the width of the channel and has crests and troughs aligned at roughly $45^{\circ}$ to the walls, like the one shown in figure $1(b)$.

The stabilizing influence of buoyancy is dominant at long wavelengths, and acts primarily to reduce the wavelength of the fastest-growing mode. Surprisingly, surface tension has the same effect, and in addition stabilizes long waves but not short ones! This counterintuitive result seems to contradict the fact that surface tension penalizes the curvature of an interface, which scales as $\lambda^{-2}$. However, the membrane force in (2.1a) also scales as $\lambda^{-2}$, which implies that if it exceeds surface tension for some wavelength (as it must if buckling is to occur) then it will do so for all wavelengths. Surface tension therefore cannot suppress short waves. At the other extreme, long waves are only weakly aligned with the compressional axis of the background shear, and so even the small effect of surface tension at such wavelengths is sufficient to suppress them.

A striking change of behaviour occurs for perturbation wavelengths below a critical value $\approx 0.3-0.8$ times the channel width (depending on the aspect ratio and the surface tension). The two fastest growing stationary modes then join to form a 
pair of travelling waves with complex conjugate growth rates, and which become progressively more confined near the walls as the wavelength decreases. Both the Stokes and advection-augmented plate models capture this behaviour, whereas the simple plate model does not. However, the real part of the growth rate of these waves is much less than that of the dominant stationary mode, and it remains to be seen whether they can be observed in the laboratory.

\section{Future}

The superiority of Slim et al.'s advection-augmented thin-plate model to the classical one holds an important lesson. As the authors point out, the augmented model violates strict asymptotic consistency, because the added advection term is of higher order in the small parameter $d / L$ than all the other terms in the model equations. The model's success suggests that asymptotic consistency is like the avoidance of parallel fifths in musical composition: a worthy principle, but one to be abandoned without hesitation when higher considerations so dictate. Another example in fluid mechanics is the model of Eggers \& Dupont (1994) for capillary breakup of a viscous jet, the predictive power of which is greatly increased by use of the exact (but asymptotically inconsistent) expression for the mean curvature of the jet's surface.

The next step is clearly to compare the authors' theoretical predictions with laboratory experiments in which the vertical deflection of the plate is accurately measured. Experiments in a long rectangular channel would be ideal, but this geometry is hard to realize in the laboratory. It would probably be more practical to perform careful experiments using the traditional annular geometry (figure 1a), and then to extend the advection-augmented plate model to this case. It would also be desirable to employ a more general theory for plates with finite midsurface curvature, which would allow the amplitude (and not just the pattern) of the buckling to be predicted.

\section{References}

Benjamin, T. B. \& Mullin, T. 1988 Buckling instabilities in layers of viscous liquid subjected to shearing. J. Fluid Mech. 195, 523-540.

Buckmaster, J. D., Nachman, A. \& Ting, L. 1975 The buckling and stretching of a viscida. J. Fluid Mech. 69, 1-20.

Eggers, J. \& Dupont, T. F. 1994 Drop formation in a one-dimensional approximation of the Navier-Stokes equations. J. Fluid Mech. 262, 205-221.

Howell, P. J. 1996 Models for thin viscous sheets. Eur. J. Appl. Math. 7, 321-343.

Johnson, A. M. \& Fletcher, R. C. 1994 Folding of Viscous Layers. Columbia University Press.

LABARbera, C. H. \& MACAyeAL, D. R. 2012 Traveling supraglacial lakes on George VI Ice Shelf. Geophys. Res. Lett., doi:10.1029/2011GL049970, in press.

NiORDSON, F. I. 1985 Shell Theory. North-Holland.

RIBE, N. M. 2002 A general theory for the dynamics of thin viscous sheets. J. Fluid Mech. 457, $255-283$.

Ribe, N. M. 2003 Periodic folding of viscous sheets. Phys. Rev. E 68, 036305.

Skorobogatiy, M. \& Mahadevan, L. 2000 Folding of viscous sheets and filaments. Europhys. Lett. 52, 532-538.

Slim, A. C., Teichman, J. \& Mahadevan, L. 2012 Buckling of a thin-layer Couette flow. J. Fluid Mech. 694, 5-28.

Suleiman, S. M. \& Munson, B. R. 1981 Viscous buckling of thin fluid layers. Phys. Fluids 24 (1), $1-5$.

TAYLOR, G. I. 1969 Instability of jets, threads and sheets of viscous fluid. In 12th International Congress of Applied Mechanics (ed. M. Hétenyi \& W. G. Vincenti). pp. 382-388. Berlin, Springer-Verlag. 